\title{
Multilevel Factors Underlying Adolescent Retention and Disengagement in HIV Care
}

\author{
Nathan Risk ${ }^{1}$, Judith Toromo ${ }^{2,3}$, Chelsea Misquith, and Leslie A. Enane ${ }^{2,3}$ \\ ${ }^{1}$ Indiana University School of Medicine, ${ }^{2}$ Academic Model Providing Access To Healthcare \\ (AMPATH), Eldoret, Kenya, ${ }^{3}$ The Ryan White Center for Pediatric Infectious Disease and Global \\ Health, Department of Pediatrics, Indiana University School of Medicine
}

Background/Objective: Adolescents (ages 10-19) living with HIV are at risk for disengagement from HIV care, resulting in poor health and potential for viral transmission. It is important to uncover the central barriers that result in adolescents becoming disengaged from care in order to develop interventions or models to support adolescent retention. We sought to investigate the multilevel barriers and facilitators to adolescent retention in HIV care.

Methods: A systematic search was performed to identify relevant studies from 1994 onwards, both qualitative and quantitative, in any language, and in all geographic regions. Title and abstract screening identified relevant articles. A full text review determined final inclusion. Key variables were extracted, focused on the range of multilevel barriers and facilitators to retention, and any differences according to population or setting. Preliminary findings are summarized.

Results: Quantitative factors associated with disengagement include: older adolescence, racial or ethnic minority status, family-level poverty, and receiving care at a rural clinic. Qualitative barriers to retention include: distance to the clinic and associated travel costs, psychosocial challenges and lack of support for them, anticipated stigma with inadvertent disclosure of HIV status, nondisclosure of HIV status to both the adolescent and family members, negative experiences with care providers, challenges requesting absence from school, and negative school environments. Facilitators to retention include: having strong social support, having a family member with HIV, having positive relationships with providers and clinic staff, attending an adolescent-dedicated clinic or a clinic with adolescent-dedicated hours, having access to intensive counseling, and having access to peer groups/peer mentors.

Conclusion and Policy Implications: Improving adolescent retention requires addressing the multilevel factors that lead to disengagement. Interventions with potential to mitigate against the range of challenges adolescents face are needed to support adolescents to overcome these challenges and continue in life-long HIV care. 